\section{Formula for selecting quality in-office lab equipment}

\section{To the Editor:}

Passage of the "Clinical Laboratory Improvement Amendment of 1988" (Public Law 100-578) on Oct 31, 1988 , means the federal government is intruding, once more, on how we physicians operate our offices. I, for one, welcome such "interference." With specific accreditation standards for in-office laboratories, this law provides long-overdue, uniform criteria to ensure high performance levels. It should cause all of us physicians to reevaluate our present office laboratories.

If it's time to upgrade equipment, a word to the wise: The cost of quality varies considerably among the different in-office laboratory systems found in today's medical marketplace. Based on personal experience, my partners and I developed a formula for choosing quality equipment.

For starters, we insist that equipment manufacturers provide free, noobligation demonstrations on the premises. Training should last a minimum of 2 to 4 weeks. Of course, we pay for the reagents. In return, we receive a free training session on each piece of equipment for our clinical staff.

We now have a state-of-the-art office chemistry profile analyzer backed by a reputable company with excellent support services. We believe a profile system offers tremendous savings in reagent expense and staff time. Meanwhile, a single testing unit provides quick, accurate test results for frequently ordered profiles such as blood glucose, BUN, and potassium. Having separate single profile and multiprofile units also reduces downtime in laboratory services.
Although the variety of available in-office laboratory tests is expanding slowly, we still use a reference laboratory for more sophisticated tests not yet available for office equipment. When possible, we do repeat split-specimen testing between our office and hospital laboratories. Fortunately, the results have been well in line with each other.

Another piece of advice: Don't be fooled by the basic equipment price. Always question the sales representative about reagent expense. Over time these expenses will far outstrip the original equipment cost. Calibration materials, proficiency studies, and quality control materials add to the expense, too. Similarly, singletest units are generally more expensive to operate than reputable profile analyzers.

We cut reagent costs by using multiple-chemistry analyzers and reagent rotors. Some single-testing units no longer require repetitive calibration procedures. In our case, our profile equipment company provides quality control materials for free.

To project quality control costs, use this formula:

Number of available chemistry profiles $x$ days used per month $\times$ average reagent cost $=$ monthly reagent expense $\times$ $12=$ annual reagent expense

Annual reagent expense + annual calibration materials expense $=$ annual quality control expenditure

Depending on the model, a 12 chemistry profile analyzer costs between $\$ 2120$ and $\$ 6780$ per year for quality control materials alone. Add that expense to the actual price of the instrument, and one such machine may cost $\$ 25,500$ to $\$ 50,680$ during a 5 -year period. A yearly service contract costs approximately $\$ 1250$, a small price to pay for peace of mind.

Savings in physician and staff time make this a worthwhile purchase. The patient's printed profile is ready by the visit's end. This saves follow-up phone calls. More importantly, it enables us to inform our patients promptly of test results and enhances timely treatment decisions.

Besides increasing revenue for our practice, our new office laboratory system offers our patients a more complete medical service. Our clinical staff also enjoys the challenge of operating the equipment. The director of our hospital clinical laboratory brings us up-to-date information on maintaining quality control documentation and a federally accepted register of laboratory tests.

Government "interference" and physician consumer savvy seem a fair price to pay for all-around firstclass laboratory service.

IRVING M. GORDON, DO

President

Perry Family Practice Center, Inc Massillon, Ohio 


\section{The new starting dose... for your newly diagnosed mild to moderate hypertensives}

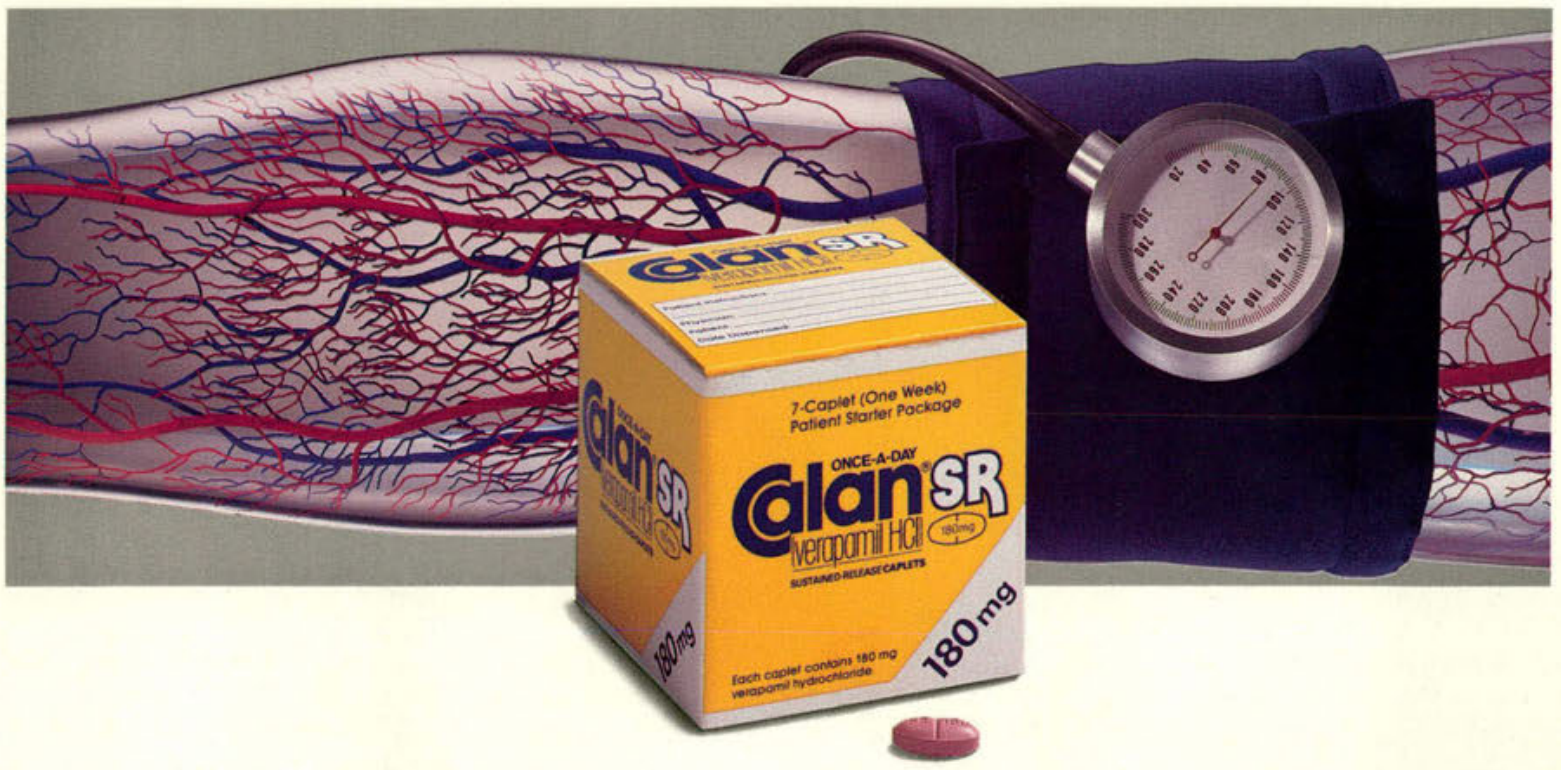

\section{NEW RECOMMENDED STARTING DOSE... CALAN SR $180 \mathrm{mg}$ q.d*}

\section{Single-agent success with Calan SR}

- $80 \%$ single-agent success rate ${ }^{\text {t1- } 6}$

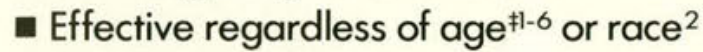
- No adverse effect on lipid profile ${ }^{7}$ - Not associated with adverse effects on serum glucose in diabetic hypertensive patients $^{8}$

- Not characteristically associated with diminished cardiac output 9

- Not associated with adverse renal effects $^{9}$

- No adverse effect on magnesium and potassium levels

- Backed by the Searle Patient Promise ${ }^{\circ}$ Program

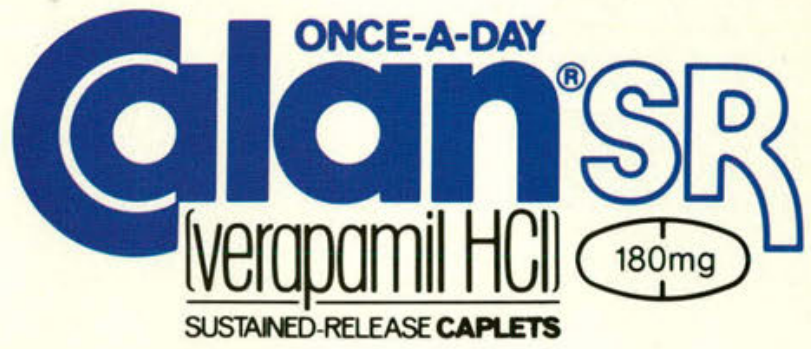

New recommended initial dose for mild to moderate hypertension

- Lower initial doses of $120 \mathrm{mg}$ a day may be warranted in patients who may have an increased response to verapamil (eg, the elderly or small people).

tDefined by the single-agent success rate of patients achieving goal BP reduction in clinical studies -0 of over 4,000 adult patients, employing varied dosage titration schedules up to $360 \mathrm{mg}$ or $480 \mathrm{mg}$ daily.

'For adult hypertensives only. 


\section{For the newly diagnosed hypertensive...}

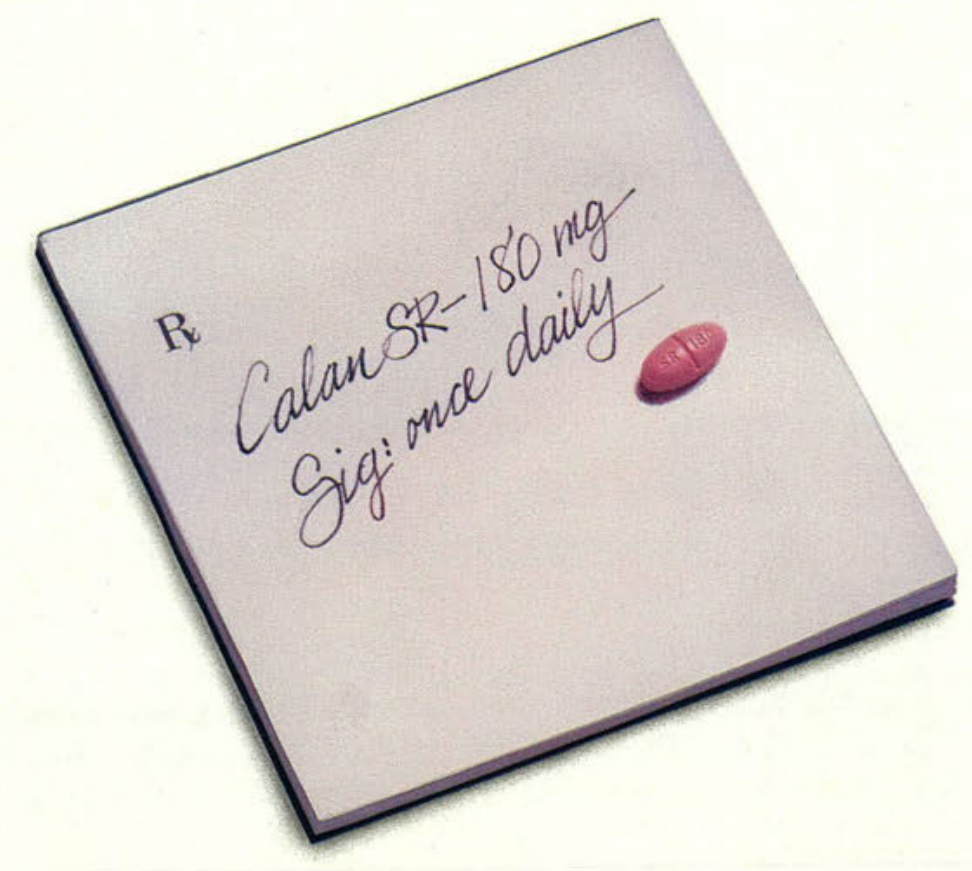

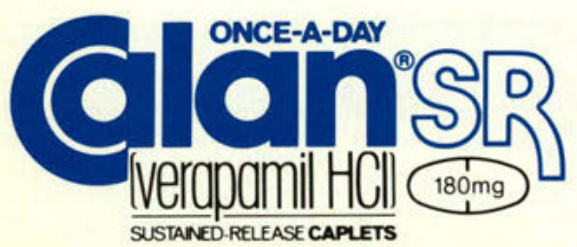

\section{BRIEF SUMMARY}

Contraindications: Severe LV dysfunction (see Warnings), hypotension (systolic pressure $<90 \mathrm{~mm} \mathrm{Hg}$ ) or cardiogenic shock, sick sinus syndrome (if no pacemaker is present), 2ndor 3rd-degree AV block (if no pacemaker is present), atrial flutter/fibrillation with an accessory bypass tract (eg, WPW or LGL syndromes), hypersensitivity to verapamil.

Warnings: Verapamil should be avoided in patients with severe LV dysfunction (eg, ejection fraction $<30 \%$ ) or moderate to severe symptoms of cardiac failure and in patients with any degree of ventricular dysfunction if they are receiving a beta-blocker. Control milder heart failure with optimum digitalization and/or diuretics before Calan SR is used. Verapamil may occasionally produce hypotension. Elevations of liver enzymes have been reported. Several cases have been demonstrated to be produced by verapamil. Periodic monitoring of liver function in patients on verapamil is prudent. Some patients with paroxysmal and/or chronic atrial flutter/fibrillation and an accessory AV pathway (eg, WPW or LGL syndromes) have developed an increased antegrade conduction across the accessory pathway bypassing the AV node, producing a very rapid ventricular response or ventricular fibrillation after receiving I.V. verapamil (or digitalis). Because of this risk, oral verapamil is contraindicated in such patients. AV block may occur (2nd- and 3rd-degree, 0.8\%). Development of marked 1stdegree block or progression to 2 nd- or 3rd-degree block requires reduction in dosage or rarely, discontinuation and institution of appropriate therapy. Sinus bradycardia, 2nd-degree $\mathrm{AV}$ block, sinus arrest, pulmonary edema and/or severe hypotension were seen in some critically ill patients with hypertrophic cardiomyopathy who were treated with verapamil. Precautions: Verapamil should be given cautiously to patients with impaired hepatic function (in severe dysfunction use about $30 \%$ of the normal dose) or impaired renal function, and patients should be monitored for abnormal prolongation of the PR interval or other signs of overdosage. Verapamil may decrease neuromuscular transmission in patients with Duchenne's muscular dystrophy and may prolong recovery from the neuromuscular blocking agent vecuronium. It may be necessary to decrease verapamil dosage in patients with attenuated neuromuscular transmission. Combined therapy with beta-adrenergic blockers and verapami may result in additive negative effects on heart rate, atrioventricular conduction and/or cardiac contractility; there have been reports of excessive bradycardia and AV block, including complete heart block. The risks of such combined therapy may outweigh the benefits. The combination should be used only with caution and close monitoring. Decreased metoprolol clearance may occur with combined use. Chronic verapamil treatment can increase serum digoxin levels by $50 \%$ to $75 \%$ during the first week of therapy, which can result in digitalis toxicity. In patients with hepatic cirrhosis, verapamil may reduce total body clearance and extrarenal clearance of digitoxin. The digoxin dose should be reduced when verapamil is given, and the patient carefully monitored. Verapamil will usually have an additive effect in patients receiving blood-pressure-lowering agents. Disopyramide should not be given within 48 hours before or 24 hours after verapamil administration. Concomitant use of flecainide and verapamil may have additive effects on myocardial contractility, AV conduction, and repolarization. Combined verapamil and quinidine therapy in patients with hypertrophic cardiomyopathy should be avoided, since significant hypotension may result. Concomitant use of lithium and verapamil may result in a lowering of serum lithium levels or increased sensitivity to lithium. Patients receiving both drugs must be monitored carefully. Verapamil may increase carbamazepine concentrations during combined use. Rifampin may reduce verapamil bioavailability. Phenobarbital may increase verapamil clearance. Verapamil may increase serum levels of cyclosporin. Concomitant use of inhalation anesthetics and calcium antagonists needs careful titration to avoid excessive cardiovascular depression. Verapamil may potentiate the activity of neuromuscular blocking agents (curare-like and depolarizing); dosage reduction may be required. Adequate animal carcinogenicity studies have not been performed. One study in rats did not suggest a tumorigenic potential, and verapamil was not mutagenic in the Ames test. Pregnancy Category C. There are no adequate and well-controlled studies in pregnant women. This drug should be used during pregnancy, labor, and delivery only if clearly needed. Verapamil is excreted in breast milk; therefore, nursing should be discontinued during verapamil use.

Adverse Reactions: Constipation (7.3\%), dizziness (3.3\%), nausea $(2.7 \%)$, hypotension $(2.5 \%)$, headache $(2.2 \%)$, edema $(1.9 \%)$, CHF, pulmonary edema $(1.8 \%)$, fatigue $(1.7 \%)$, dyspnea (1.4\%), bradycardia: $\operatorname{HR}<50 / \mathrm{min}(1.4 \%)$, AV block: total $1^{\circ}, 2^{\circ}, 3^{\circ}(1.2 \%), 2^{\circ}$ and $3^{\circ}(0.8 \%)$, rash $(1.2 \%)$, flushing $(0.6 \%)$, elevated liver enzymes. The following reactions, reported in $1.0 \%$ or less of patients, occurred under conditions where a causal relationship is uncertain: angina pectoris, atrioventricular dissociation, chest pain, claudication, myocardial infarction, palpitations, purpura (vasculitis), syncope, diarrhea, dry mouth, gastrointestinal distress, gingival hyperplasia, ecchymosis or bruising, cerebrovascular accident, confusion, equilibrium disorders, insomnia, muscle cramps, paresthesia, psychotic symptoms, shakiness, somnolence, arthralgia and rash, exanthema, hair loss, hyperkeratosis, macules, sweating, urticaria, Stevens-Johnson syndrome, erythema multiforme, blurred vision, gynecomastia, increased urination, spotty menstruation, impotence. $12 / 21 / 89 \cdot P 90-W 198 \mathrm{~V}$

References: 1. Reinfrank J, Eckardt A, Schneider G, et al: Long-term efficacy and safety of verapamil SR $240 \mathrm{mg}$ in hypertension. Acta Therapeutica 1989:15:221-235. 2. Saunders E, Weir MR, Kong BW, et al: A comparison of the efficacy and safety of a beta blocker, calcium channel blocker, and converting enzyme inhibitor in hypertensive blacks. Arch Intern Med laccepted for publication) 3. Speders S, Sosna J, Schumacher A, et al: Efficacy and safety of verapamil SR $240 \mathrm{mg}$ in essentia hypertension: Results of a multicentric phase IV study. J Cardiovasc Pharmacol 1989;13(suppl 4):S47S49. 4. Henry JA, Chester PC, Latham AN: Sustained-release verapamil or atenolol in essentia hypertension. J Drug Dev 1988:1:69-75, 5. Midtbo KA, Hals O, Lauve O: A new sustained-release formulation of verapamil in the treatment of hypertension. J Clin Hypertens 1986:3:125S-132S 6. Bochsler JA, Simmons RL, Ward PJ, et al: Verapamil SR and propranolol LA: A comparison of efficacy and side effects in the treatment of mild to moderate hypertension. $J$ Hum Hypertens 1988;: :305-310. 7. Midtbø K, Lauve O, Hals O: No metabolic side effects of long-term treatmen with verapamil in hypertension. Angiology 1988;39:1025-1029. 8. Cruickshank JK, Anderson NMcF, Wadsworth J, ef al: Treating hypertensive diabetics: A comparison of verapamil and metoprolol in black and white patients. J Cardiovasc Pharmacol 1987;10(suppl 10):S85-S86. 9. Schmieder RE, Messerli FH, Garavaglia GE, et al: Cardiovascular effects of verapamil in patients with essential hypertension. Circulation 1987;75:1030-1036.

A90-W259T

Address medical inquiries to: G.D. Searle \& Co Medical \& Scientific Information Department
4901 Searle Parkway

Skokie, IL 60077 